\title{
Evaluation of glandular dose in mammography in presence of breast cysts using Monte Carlo simulation
}

\author{
Mohammad Reza DeEVBAND ${ }^{\mathrm{a}}$, Zeinab KAVEH ${ }^{\mathrm{a}}$, Mahdi GHORBANI ${ }^{\mathrm{a},{ }^{*}}$, Benyamin KHAJETASH $^{\mathrm{b}}$ \\ ${ }^{a}$ Biomedical Engineering and Medical Physics Department, School of Medicine, Shahid Beheshti University of Medical \\ Sciences, Tehran, Iran \\ ${ }^{b}$ Medical Physics Department, School of Medicine, Iran University of Medical Sciences, Tehran \\ *E-mail address: mhdghorbani@gmail.com
}

\begin{abstract}
Background: Normalized glandular dose (DgN) is an important dosimetric quantity in mammography.

Aim: In this study, the effect of the presence of breast cysts and their size, number and location on $\mathrm{DgN}$ is evaluated. Materials and methods: The effect of the presence of cysts in breast was examined using MCNPX code. This was performed by taking homogeneous breast phantoms containing spheroid breast cysts into account. The radius of the cysts, numbers of the cysts, and depth of the cysts, and their location were variable. Various electron energies were also considered. Finally, these results were compared with the results of a cyst-less breast phantom.

Results: The results show that the effect of the presence of cysts in the breast depends on the size, number and location of cysts. The presence of cysts at lower depths leads to a decrease in the DgN values, compared to the breast phantom without cysts. The presence of cysts in the breast phantom has an effect of -7 to +14 percent on the DgN values under the conditions considered in this modeling. This effect is independent of the X-ray tube voltage, the breast phantom thickness, and glandular ratio, and depends only on the number and size and location of the cysts. The bigger radius and number of cysts, the greater effect on $\mathrm{DgN}$ value.
\end{abstract}

Key words: mammography; Monte Carlo simulation; normalized glandular dose; cysts; dosimetry.

\section{Introduction}

Breast cancer is the most common cancer in women, affecting 2.1 million women annually and causing the highest number of cancer deaths among women in the world. ${ }^{1}$ In 2018, 627,000 women are estimated to have died due to breast cancer which is accounted for 15 percent of all cancer deaths among women. ${ }^{1}$ While the rate of breast cancer among women in developed countriesis higher, the rate is increasing worldwide in almost all countries. Early detection is critical to improve breast cancer outcomes and survival. Various methods, including mammography, clinical breast examination and breast selfexamination, are accounted as the breast cancer screening tools. In mammography low energy X-rays are used to detect breast abnormalities. It was shown that the mortality rate of breast cancer was reduced by about $20 \%$ by using mammography. ${ }^{1}$ Small changes in the composition of breast tissue can be detected with this technique.

Like other medical imaging techniques using X-rays, there is always a small risk of development of cancer due to the use of ionization radiation. Therefore, it is important to evaluate the dose given to a patient during mammography. ${ }^{2}$ In other words, the as low as reasonably achievable (ALARA) principle should be taken into account in mammography. ${ }^{3}$
A number of researchers have performed precise breast dosimetry during mammography. ${ }^{4,5}$ The dose to breast tissue is estimated by patient dosimetry or, in most cases, by inphantom measurements. The breast dose depends on the X-ray spectrum (which changes with a different target and filter combination), the breast composition (the ratio of the glandular tissue to the adipose tissue), the breast thickness and the X-ray tube voltage of the mammography machine. ${ }^{6}$

It is generally accepted that glandular tissue in breast is the most sensitive tissue to radiation. ${ }^{7-9}$ It was, therefore, widely accepted that the average glandular dose (AGD) is the most suitable quantity to estimate the risk of radiation-induced cancer. Several calculation methods have been introduced for the calculation of the deposited energy in the breast. The majority of these methods are based on Monte Carlo simulation, which is used to calculate the surface dose or air kerma conversion factor to the effective dose on the phantom surface. This is called the normalized glandular dose $(\mathrm{DgN})$ conversion factor. This conversion factor is related to parameters such as the composition of anode and filter type, breast tissue composition, breast tissue thickness, X-ray tube voltage and X-ray beam quality. In many studies, the effect of these factors on the glandular dose was investigated. ${ }^{9-11}$ 
Breast tissue consists of adipose and gland. The ratio of the gland to adipose in the breast varies among different women. These differences in the structure of the breast affect the radiation dose to the breast. About one-third of women aged 35 to 50 years have breast cysts. ${ }^{12}$ Breast cysts produce heterogeneity in the breast tissue, which can affect the average glandular dose. Despite the high prevalence of this disease in women, to the best of our knowledge, the effect of the presence of cysts on breast dose in mammography has not been studied yet.

Many studies have been conducted on the factors affecting the doses received in mammography, but in most studies, breast tissue has been considered as a homogeneous medium. ${ }^{4,11}$ In some studies, heterogeneity of breast tissue has been considered. ${ }^{14-17}$

The aim of this study is to evaluate the effect of the presence of breast cysts to $\mathrm{DgN}$ with changes in the thickness, the glandular composition of the breast tissue and the voltage of the X-ray tube. Finally, the results will be compared with the situation where homogeneous breast tissue is considered.

\section{Materials and methods}

Initially, the mammography unit was simulated using the manufacturer's geometry data and the simulation was validated. Then, the effect of different parameters (such as depth of cysts, $\mathrm{X}$-ray tube voltage, glandular ratio, and breast thickness) on the breast dose was investigated. MCNPX Monte Carlo code was used for the simulations. The densities and compositions of materials should be defined in MCNPX program. Therefore, the breast phantom was defined as a phantom containing homogeneous distribution in the form of atomic fractions of glandular tissue and adipose. The skin was also defined at the surface of phantom with $4 \mathrm{~mm}$ thickness. The densities of different materials were also defined. In the case of the definition of cysts, they were defined as spheres containing cyst liquid.

\section{X-ray tube simulation}

In the current study, the MCNPX code (version 2.6.0) was used to simulate the PMAM 100-F mammography machine (Payamed company, Tehran, Iran). ${ }^{18-19}$

The source was assumed to be a surface source of $0.3 \times 0.3$ $\mathrm{mm}^{2}$ area, from which the electrons were exited in the same direction so that the electrons were directed toward the target. The 26, 28 and $30 \mathrm{keV}$ electron energies were simulated.

To define the molybdenum target volume (anode), 6 planes were used. The target angle was assumed to be 15 degrees. The exit window of the X-ray tube was 0.5-millimeter thickness of beryllium. The extra filter (of molybdenum) had 30 micrometers thickness. The collimator of the unit was assembled into 4 jaws of lead with thickness of $2 \mathrm{~mm}$. The opening of the collimators was such that, a $24 \times 18 \mathrm{~cm}^{2}$ field was created on the film surface at source to image distance
(SID) of $65 \mathrm{~cm}$. A schematic view of the simulated X-ray tube is presented in Figure 1.

The X-ray spectrum from the Monte Carlo simulation was compared with the spectrum reported by the Institute of Physics and Engineering in Medicine (IPEM) ${ }^{20}$ by statistical analysis (by SPSS software, version 16.0). Paired $t$-test was used for this purpose. An acceptable discrepancy of 5\% (also referred to as a significant level) was selected.

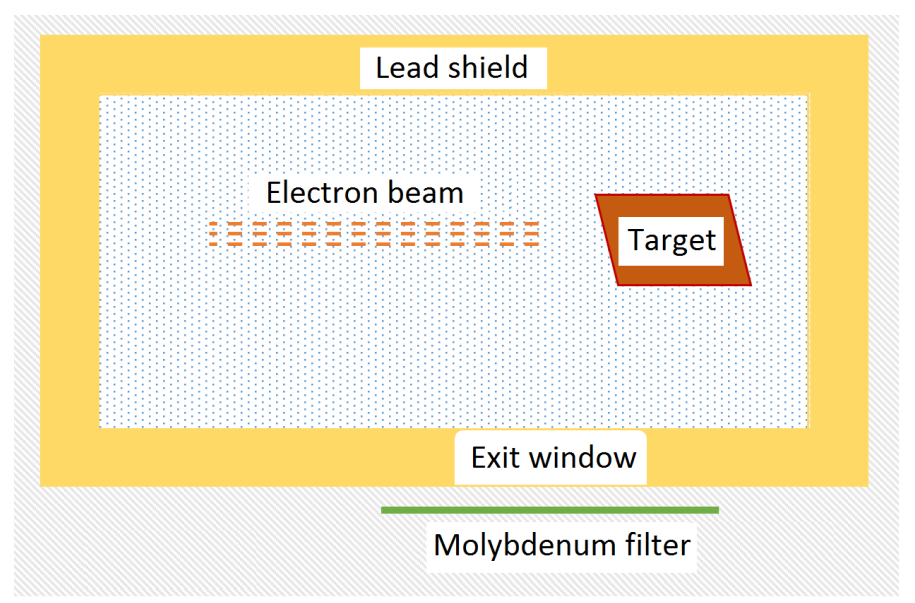

Figure 1. A schematic view of the simulated X-ray tube

To calculate the percentage depth dose (PDD) using MCNPX, a cubic phantom was considered at a distance of $65 \mathrm{~cm}$ from the source. The energy of the simulated electron was $30 \mathrm{keV}$ which is equivalent to a voltage of $30 \mathrm{kV}$. The phantom dimensions were $10 \times 10 \times 4 \mathrm{~cm}^{3}$. The dose was calculated at the surface of the phantom and depths of 1,2, 3 and $4 \mathrm{~cm}$ in spherical cells with $0.5 \mathrm{~mm}$ radius. The calculation of the dose was performed in two steps by the MCNPX code. In the first step, energy fluence was calculated using *F5 tally. In the second step, the fluence-to-dose conversion factor was used to calculate the dose. In fact, these conversion factors are mass energy absorption coefficients for the phantom constituent material (perspex). The mass-energy absorption coefficients for different elements and compositions of materials are available in the literature. ${ }^{21}$

To measure the PDD, 4 slabs of perspex with dimensions of $10 \times 10 \mathrm{~cm}^{2}$ and thickness of $1 \mathrm{~cm}$ were used. A Barracuda solid-state detector (RTI Electronic manufacturing Co.) was used to measure the dose. The aforementioned mammography machine is used in Baharloo Hospital (Tehran, Iran). The exposure conditions of the X-ray tube were chosen to be 15 $\mathrm{mAs}$ and $30 \mathrm{kV}$. Then the irradiation was performed and the dose in the phantom was recorded in $\mathrm{mGy}$. Measurements were performed for all depths (1, 2, 3 and $4 \mathrm{~cm}$ ). Finally, the PDD curve was plotted in Microsoft Excel software (version 13.0.0) for the calculated and measured values, and the percentage difference between these two data series was investigated. 


\section{Phantom simulation}

The simulation of breast phantom was performed in three steps: 1-homogenous breast phantom without cyst, 2homogeneous breast phantom in the presence of cysts and 3heterogeneous breast phantom. In each step, the factors influencing on the calculation of the AGD (such as the thickness of the breast phantom, the glandular ratio of the breast, the energy of the photons, depth of cysts, cysts size, and number of cysts) were changed and their effect on the AGD was evaluated.

\section{Homogeneous breast phantom without cysts}

Similar to other dosimetric studies in mammography, breast phantom was considered as a homogeneous mixture of glandular and adipose tissue and the densities and atomic compositions are based on previous studies in this field. ${ }^{16-17,22-}$

${ }^{23}$ The distance of the surface of the phantom to the source was $65 \mathrm{~cm}$ and the electrons energies of 26,30 , and $32 \mathrm{keV}$ were investigated. The compressor plate was considered as a 3 millimeters thick plate made of polycarbonate with a dimension of $24 \mathrm{~cm} \times 18 \mathrm{~cm}$, which was located on the breast phantom. The glandular ratio of the breast phantom was evaluated as a variable. The glandular ratio of the breast was 0 , $20,50,80,100 \%$. The glandular ratio is defined as the ratio of the mass of the glandular tissue to the total breast tissue. The homogeneous breast phantom was considered as a rectangular cube with dimensions of $8 \mathrm{~cm} \times 12 \mathrm{~cm}$ and heights of 3, 5, 6,7 and $8 \mathrm{~cm}$. The heights indicate various thickness for the breast. In this phantom, similar to the other studies, the thickness of the skin was considered to be $4 \mathrm{~mm} .{ }^{4}$ The selected conditions for the phantom used for the calculation of the average glandular dose are presented in Table 1.

\section{Homogeneous breast phantom with presence of cysts}

Breast cysts were considered in the form of spheres containing cyst liquid. The density and weight percentage of the cyst elements are available in the study by Fredenberg, et al. ${ }^{24}$ The dimensions of these cysts were variable, and at each step, the radius of these cysts was increased to determine the effect of increasing the size of the cysts on the average glandular dose. The radius of the cysts was assumed to be $0.6,0.9$ and $1.2 \mathrm{~cm}$. These cysts were located at different depths in the breast tissue, so that the effect of the location of cysts on the average glandular dose can be evaluated. The depths of the cysts were considered to be $3.1,3.6$ and $5.9 \mathrm{~cm}$. These cysts were also considered in breast phantoms with different glandular percentages $(20,50$ and $80 \%)$ to evaluate the effect of the breast glandular ratio. The effect of X-ray tube voltage was also investigated. For this purpose, the values of 26,30 and 32 $\mathrm{kV}$ were selected. These variables are presented in Table 2. In Figure 2, the characteristics of the simulated breast phantom, which were used to calculate the AGD, are shown.
Table 1. Parameters of the simulated phantoms in the homogeneous phantom without cyst for calculation of AGD

\begin{tabular}{cccc}
\hline \hline Phantom number & Thickness $(\mathbf{c m})$ & Glandular ratio & Voltage $(\mathbf{k V})$ \\
\hline 1 & 3 & 20 & 30 \\
2 & 5 & 20 & 30 \\
3 & 6 & 20 & 30 \\
4 & 7 & 20 & 30 \\
5 & 8 & 20 & 30 \\
6 & 8 & 20 & 26 \\
7 & 8 & 50 & 26 \\
8 & 8 & 80 & 26 \\
9 & 8 & 20 & 32 \\
\hline \hline
\end{tabular}

Table 2. Parameters of the simulated homogeneous phantoms for calculation of AGD in the presence of cysts

\begin{tabular}{|c|c|c|c|c|c|c|}
\hline $\begin{array}{l}\text { Phantom } \\
\text { number }\end{array}$ & $\begin{array}{l}\text { Thickness } \\
\text { (cm) }\end{array}$ & $\begin{array}{c}\text { Glandular } \\
\text { ratio }\end{array}$ & $\begin{array}{c}\text { Voltage } \\
(\mathbf{k V})\end{array}$ & $\begin{array}{l}\text { Number } \\
\text { of cysts }\end{array}$ & $\begin{array}{c}\text { Cysts } \\
\text { radius } \\
(\mathrm{cm})\end{array}$ & $\begin{array}{c}\text { Depth } \\
\text { of cysts } \\
\text { (cm) }\end{array}$ \\
\hline 1 & 8 & 20 & 30 & 6 & 1.2 & 1.3 \\
\hline 2 & 8 & 20 & 30 & 6 & 1.2 & 3.6 \\
\hline 2 & 8 & 20 & 30 & 6 & 1.2 & 5.9 \\
\hline 4 & 8 & 20 & 26 & 6 & 1.2 & 1.3 \\
\hline 5 & 8 & 20 & 32 & 6 & 1.2 & 1.3 \\
\hline 6 & 8 & 20 & 26 & 6 & 0.9 & 5.9 \\
\hline 7 & 8 & 20 & 32 & 6 & 0.6 & 5.9 \\
\hline 8 & 8 & 50 & 26 & 6 & 1.2 & 1.3 \\
\hline 9 & 8 & 80 & 26 & 6 & 1.2 & 1.3 \\
\hline 10 & 8 & 50 & 26 & 6 & 1.2 & 5.9 \\
\hline 11 & 8 & 80 & 26 & 6 & 1.2 & 5.9 \\
\hline 12 & 8 & 20 & 30 & 6 & 0.9 & 1.3 \\
\hline 13 & 7 & 20 & 30 & 6 & 0.9 & 1.3 \\
\hline 14 & 6 & 20 & 30 & 6 & 0.9 & 1.3 \\
\hline 15 & 8 & 20 & 30 & 6 & 0.9 & 5.9 \\
\hline 16 & 7 & 20 & 30 & 6 & 0.9 & 5.9 \\
\hline 17 & 6 & 20 & 30 & 6 & 0.9 & 5.9 \\
\hline 18 & 8 & 20 & 30 & 6 & 0.6 & 1.3 \\
\hline 19 & 8 & 20 & 30 & 6 & 0.6 & 5.9 \\
\hline 20 & 8 & 20 & 30 & 4 & 1.2 & 1.3 \\
\hline 21 & 8 & 20 & 30 & 2 & 1.2 & 1.3 \\
\hline 22 & 8 & 20 & 30 & 4 & 1.2 & 5.9 \\
\hline 23 & 8 & 20 & 30 & 2 & 1.2 & 5.9 \\
\hline
\end{tabular}

\section{Heterogeneous breast phantom}

As mentioned in the previous section, the breast tissue has glandular tissue and adipose, separately. To compare the results of dose calculations, in contrast to the homogeneous breast phantom, a phantom was simulated in which the tissue of the gland and adipose were separated. This phantom was assumed to have dimensions of $8 \mathrm{~cm} \times 8 \mathrm{~cm}$. In this phantom, the glandular tissue was considered as 12 spheres with a radius of 1.76 and $1.28 \mathrm{~cm}$. In this case, the glandular ratio was $50 \%$ and $20 \%$, respectively. A voltage of $30 \mathrm{kV}$ was chosen. The cyst radius, glandular ratio, thickness and voltage variables for simulation of the heterogeneous phantom are described in Table 3. 


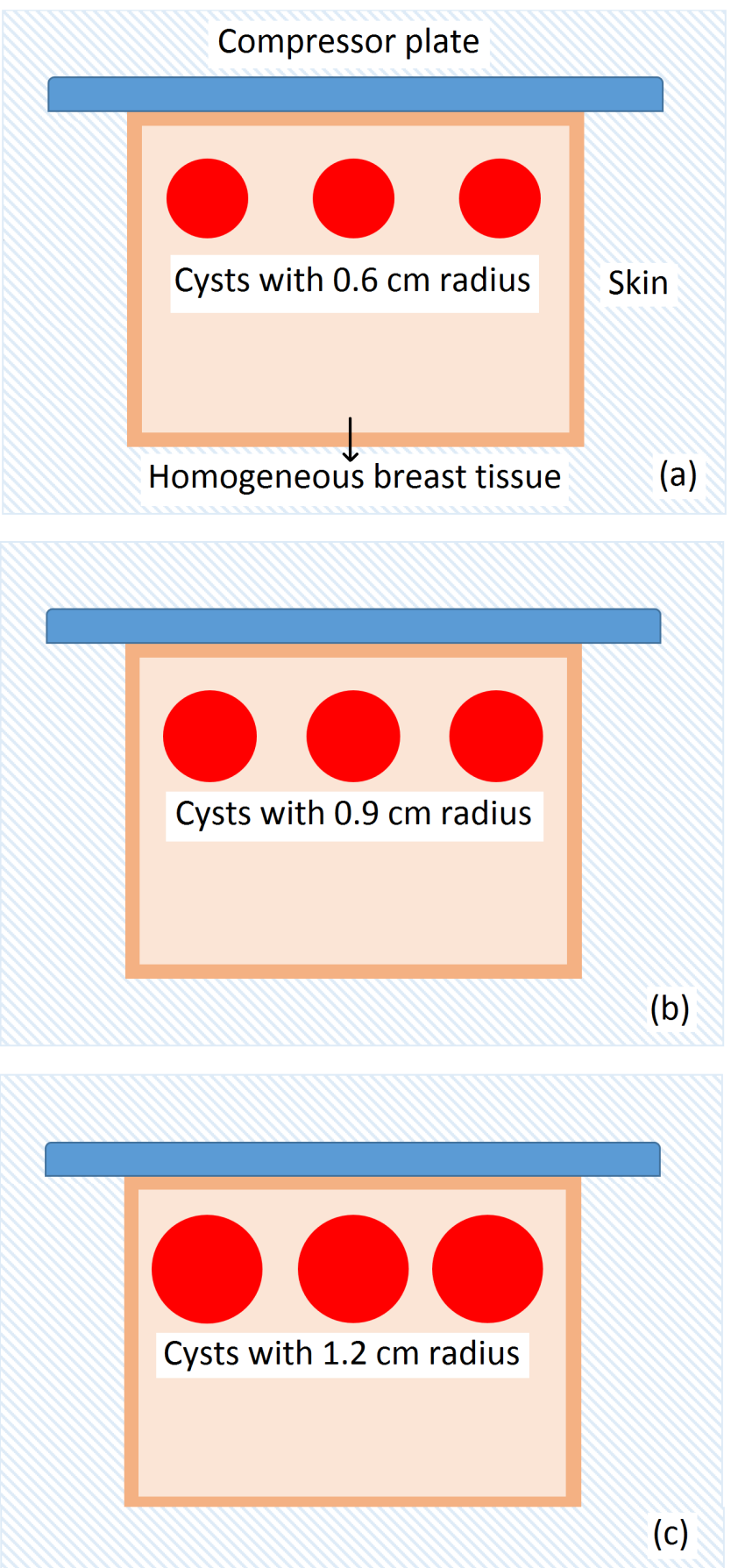

Figure 2. The breast phantom in the $y-z$ view in the presence of cyst, for different sizes of cysts: (a) the radius of the cysts is 0.6 centimeter (b) the radius of the cysts is 0.9 centimeter (c) the radius of the cysts is $\mathbf{1 . 2}$ centimeter.

Table 3. Parameters of the simulated heterogeneous phantoms for calculation of AGD

\begin{tabular}{ccccc}
\hline \hline $\begin{array}{c}\text { Phantom } \\
\text { number }\end{array}$ & $\begin{array}{c}\text { Thickness } \\
(\mathbf{c m})\end{array}$ & $\begin{array}{c}\text { Glandular } \\
\text { ratio }\end{array}$ & Voltage (kV) & $\begin{array}{c}\text { Cysts radius } \\
(\mathbf{c m})\end{array}$ \\
\hline 1 & 8 & 5 & 30 & 0.8 \\
2 & 8 & 20 & 30 & 1.28 \\
3 & 8 & 50 & 30 & 1.76 \\
4 & 8 & 100 & 30 & none \\
\hline \hline
\end{tabular}

\section{DgN calculation in homogeneous and heterogeneous breast phantoms}

Normalized glandular dose $(\mathrm{DgN})$ is derived from the average glandular dose and the Entrance Skin Air Kerma (ESAK) ${ }^{16}$ The average glandular dose and air kerma were calculated by the simulation code and the values were expressed in Gy and Roentgen, respectively. The average glandular dose depends on the composition of the breast, therefore, in both homogeneous and heterogeneous breast phantoms, the average glandular dose was calculated by the MCNPX code.

\section{Calculation of the average glandular dose in homogeneous phantom}

The total dose of the breast was calculated using the MCNPX simulation code. As it was explained in the introduction section, for calculation of the average glandular dose, only the dose to the glandular part should be considered, therefore, for calculation of the average glandular dose, the $G$ factor, which was presented in previous studies, was used. ${ }^{4}$ Since the $G$ factor depends on energy, dose in the homogenous breast tissue $(D)$ and $G$ were calculated in different energy bins and multiplied, then AGD was calculated as the sum of these products.

\section{Calculation of average glandular dose in heterogeneous phantom}

In the heterogeneous breast phantom, glandular spheres were distributed uniformly in the adipose medium. To change the glandular fraction, the radii of the glandular spheres were changed.

In this case, the average glandular dose is equal to:

$\mathrm{AGD}=\mathrm{E}_{\mathrm{T}} /\left(\mathrm{n} \times \mathrm{m}_{\mathrm{g}}\right)$

Where $E_{T}$ is the total energy deposited in the glandular tissues, $n$ is the number of glandular tissues and $m_{g}$ is the mass of the glandular tissue. The energy deposited in the glandular tissue was calculated using the MCNPX simulation code and is expressed in Gy. Similar to the previous state, at this stage, F6 tally was used.

\section{Calculation of ESAK}

To calculate the air kerma, a cube of air was placed at $1 \mathrm{~cm}$ below the compressor plate, $4 \mathrm{~cm}$ away from the chest and the air kerma was calculated in this volume using F6 tally. The output value for this tally is expressed in terms of $\mathrm{MeV} / \mathrm{g}$ in the MCNPX code. This calculation was performed for electrons of 26, 30 and $32 \mathrm{keV}$ energy, which are equivalent to 26, 30 and 32 kilovolts for the X-ray tube. According to formula 1, by dividing the average glandular dose value on the air kerma at the phantom surface, the normalized glandular dose $(\mathrm{DgN})$ was obtained. 


\section{Results}

\section{Validation of the $\mathrm{X}$-ray tube simulation}

The X-ray spectrum was investigated for 26, 28 and 30 kilovoltages. These spectra are illustrated in Figure 3a. In this study, the comparison with IPEM spectrum was performed only for the $30 \mathrm{kV}$ voltage. Figure $\mathbf{3 b}$ shows the spectra from the simulation and IPEM for the $30 \mathrm{kV}$.

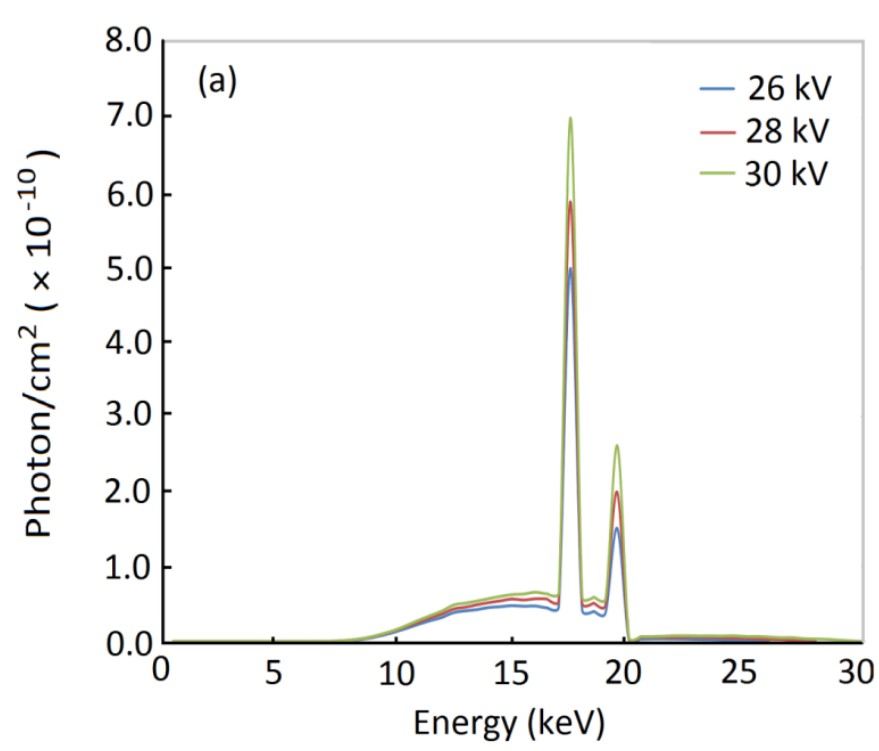

Percentage depth dose was obtained by simulation and experimental methods. Figure 4 shows the PDD obtained from Monte Carlo calculation and measurement. The maximum discrepancy observed between these two graphs is $5.2 \%$ which is related to the depth of $2 \mathrm{~cm}$.

Figure 3. (a) The X-ray spectrums for 26,28 and $30 \mathrm{keV}$ electrons (b). The X-ray spectrum derived from the simulation and the IPEM report
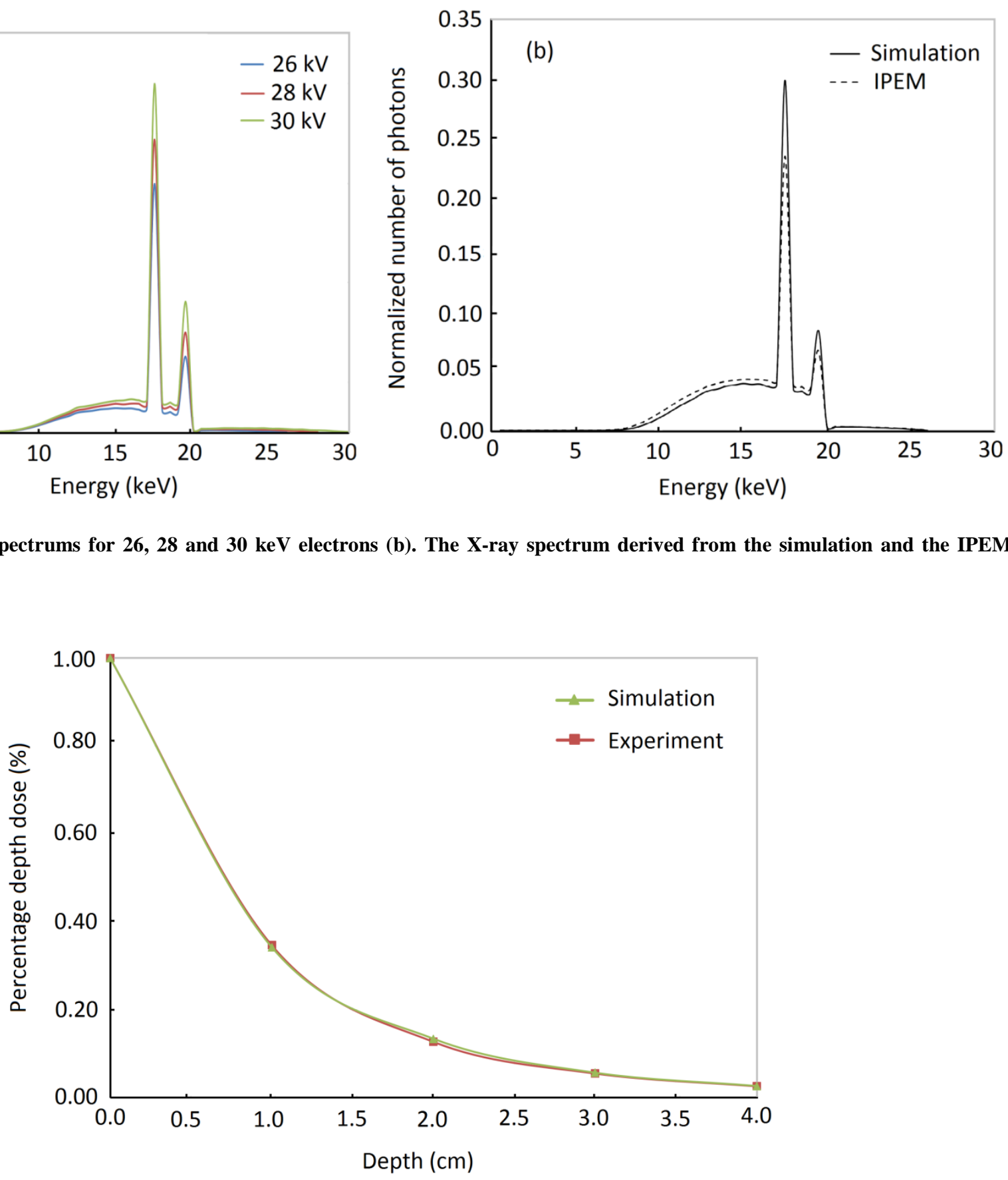

Figure 4. PDD obtained using simulation and in-phantom measurement 


\section{DgN results for homogeneous breast phantom without cysts}

Figure 5a shows DgN plot versus glandular fraction with $8 \mathrm{~cm}$ breast thickness and $30 \mathrm{kV}$ voltage in the homogeneous breast phantom. The $\mathrm{DgN}$ diagram versus breast thickness in the homogeneous breast phantom with $20 \%$ glandular ratio and 30 $\mathrm{kV}$ voltage is illustrated in Figure $\mathbf{5 b}$.

\section{DgN results for homogeneous breast phantom in the presence of cysts}

In this step, the characteristics of cysts (such as the size, the number and the depth of cysts) were changed step by step and the $\mathrm{DgN}$ results were compared with the $\mathrm{DgN}$ results from the homogeneous breast phantom in which the cysts were not present. In addition to the characteristics of the cysts, other

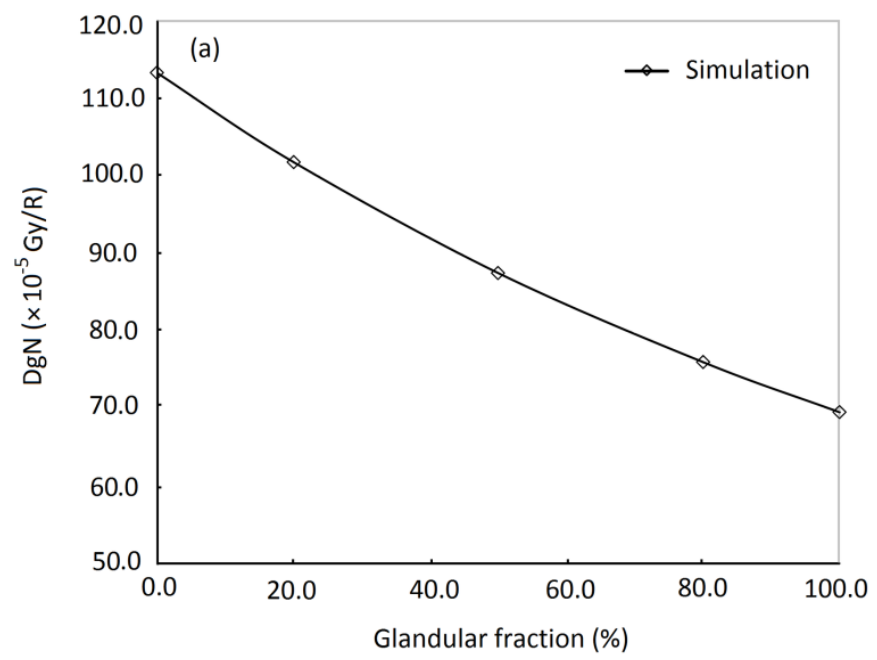

parameters affecting $\mathrm{DgN}$ (such as the thickness of the breast phantom, the glandular ratio of breast and X-ray tube voltage), in both phantoms (with and without cysts) were changed and the results were The plot of $\mathrm{DgN}$ in terms of depth of cysts in the homogeneous phantom with and without cysts is presented in Figure 6. The values of $\mathrm{DgN}$ and the relative difference between the values in the case of with and without cysts in the homogeneous breast phantom are listed in Table 4.

Table 4. DgN values and relative differences between the values in the homogeneous breast phantom with and without cysts.

\begin{tabular}{cccc}
\hline \hline $\begin{array}{c}\text { Depth of } \\
\text { cysts }\end{array}$ & $\begin{array}{c}\text { DgN (with cyst) } \\
(\mathbf{G y} / \mathbf{R})\end{array}$ & $\begin{array}{c}\text { DgN (without cyst) } \\
(\mathbf{G y} / \mathbf{R})\end{array}$ & $\begin{array}{c}\text { Relative difference } \\
(\mathbf{\%})\end{array}$ \\
\hline 1.3 & 87.3 & 107.1 & 14 \\
3.6 & 105 & 107.1 & -3 \\
5.9 & 108.96 & 107.1 & -6.8 \\
\hline \hline
\end{tabular}

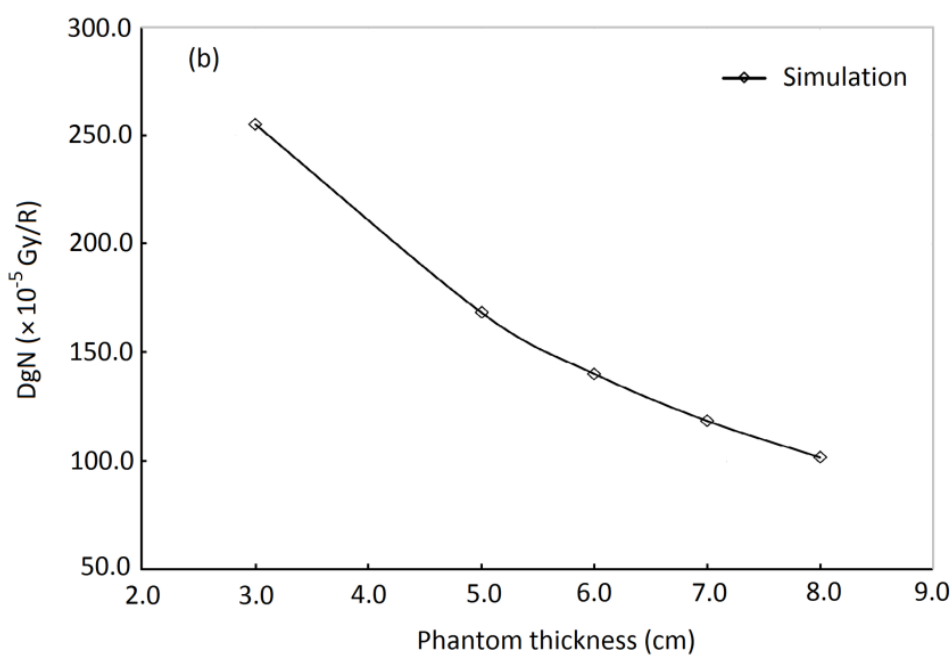

Figure 5. (a) DgN versus breast glandular fraction with $8 \mathrm{~cm}$ phantom thickness and $30 \mathrm{kV}$ voltage in the homogeneous phantom (b) DgN in terms of breast thickness in homogeneous phantom with $20 \%$ glandular ratio and $30 \mathrm{kV}$ constant voltage.

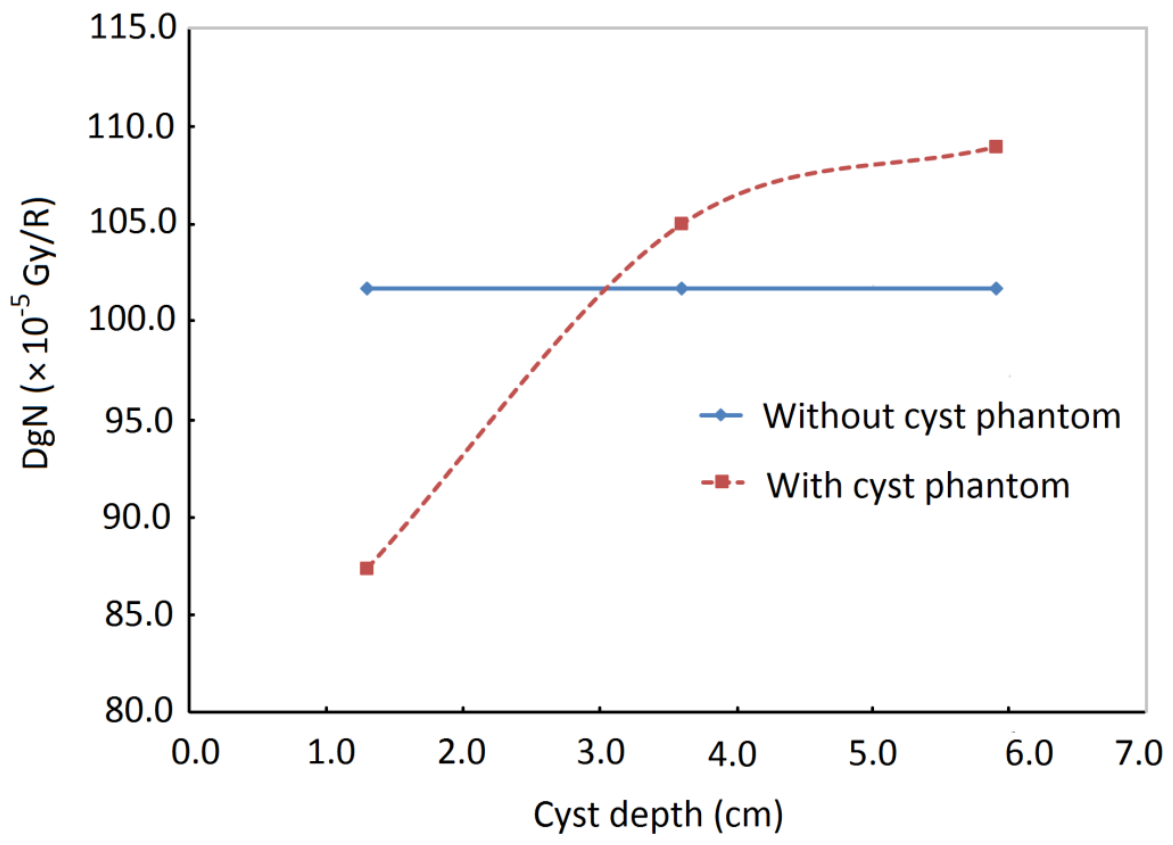

Figure 6. DgN versus depth of cysts in homogeneous breast phantom with and without cysts. 
Three breast phantoms were considered to evaluate the effect of glandular fraction on the $\mathrm{DgN}$. All three phantoms were 8 $\mathrm{cm}$ in height. The first breast phantom was considered without any cyst. In the second phantom, 6 cysts were placed at depth of $1.3 \mathrm{~cm}$, and in the third phantom, 6 cysts were placed at depth of $5.9 \mathrm{~cm}$. The glandular fractions of the phantoms were selected to be 20,50 and $80 \%$, and the results of $\mathrm{DgN}$ values are compared in Figure 7a. It should be noted that in all three cases the voltage of the X-ray tube was a constant value of 26 $\mathrm{kV}$.

Three phantoms were used to evaluate the effect of X-ray tube voltage variation on the $\mathrm{DgN}$. All the three phantoms were $8 \mathrm{~cm}$ in height. The first phantom was considered to be without cysts. The second phantom had 6 cysts at depth of $3.1 \mathrm{~cm}$, and the third phantom had 6 cysts at depth of $9.5 \mathrm{~cm}$. The calculations were performed for $26 \mathrm{kV}, 30 \mathrm{kV}$ and $32 \mathrm{kV}$ Xrays. The results of the values of $\mathrm{DgN}$ are compared in Figure 7b. It should be noted that in all these cases, the glandular fraction of the breast phantom was $20 \%$.
To evaluate the effect of breast thickness on the $\mathrm{DgN}$, three breast phantoms were considered, while all of them had a glandular fraction of $20 \%$. The first phantom was considered without any cyst. In the second breast phantom, 6 cysts were placed at depth of $3.1 \mathrm{~cm}$, and in the third phantom, 6 cysts were placed at depth of $5.9 \mathrm{~cm}$. The breast thickness (the height of the phantom) of $8 \mathrm{~cm}, 7 \mathrm{~cm}$ and $6 \mathrm{~cm}$ were evaluated. The X-ray tube voltage applied at all these steps was $30 \mathrm{kV}$ and the radius of the cysts was considered to be the constant value of $0.9 \mathrm{~cm}$. The results of the $\mathrm{DgN}$ values for these cases are compared in Figure 7c.

To evaluate the effect of the cyst size on the $\operatorname{DgN}$, three phantoms were considered, while all the three phantoms had a glandular fraction of $20 \%$ and thickness of $8 \mathrm{~cm}$. The first phantom was without any cyst. In the second phantom, 6 cysts were placed at depth of $3.1 \mathrm{~cm}$, and in the third phantom, 6 cysts were placed at depth of $5.9 \mathrm{~cm}$. Cyst radius of $0.6 \mathrm{~cm}, 0.9$ and $1.2 \mathrm{~cm}$ were assessed. The voltage applied at all the stages was $30 \mathrm{kV}$. The results of the $\mathrm{DgN}$ values for these cases are compared in Figure 7d.
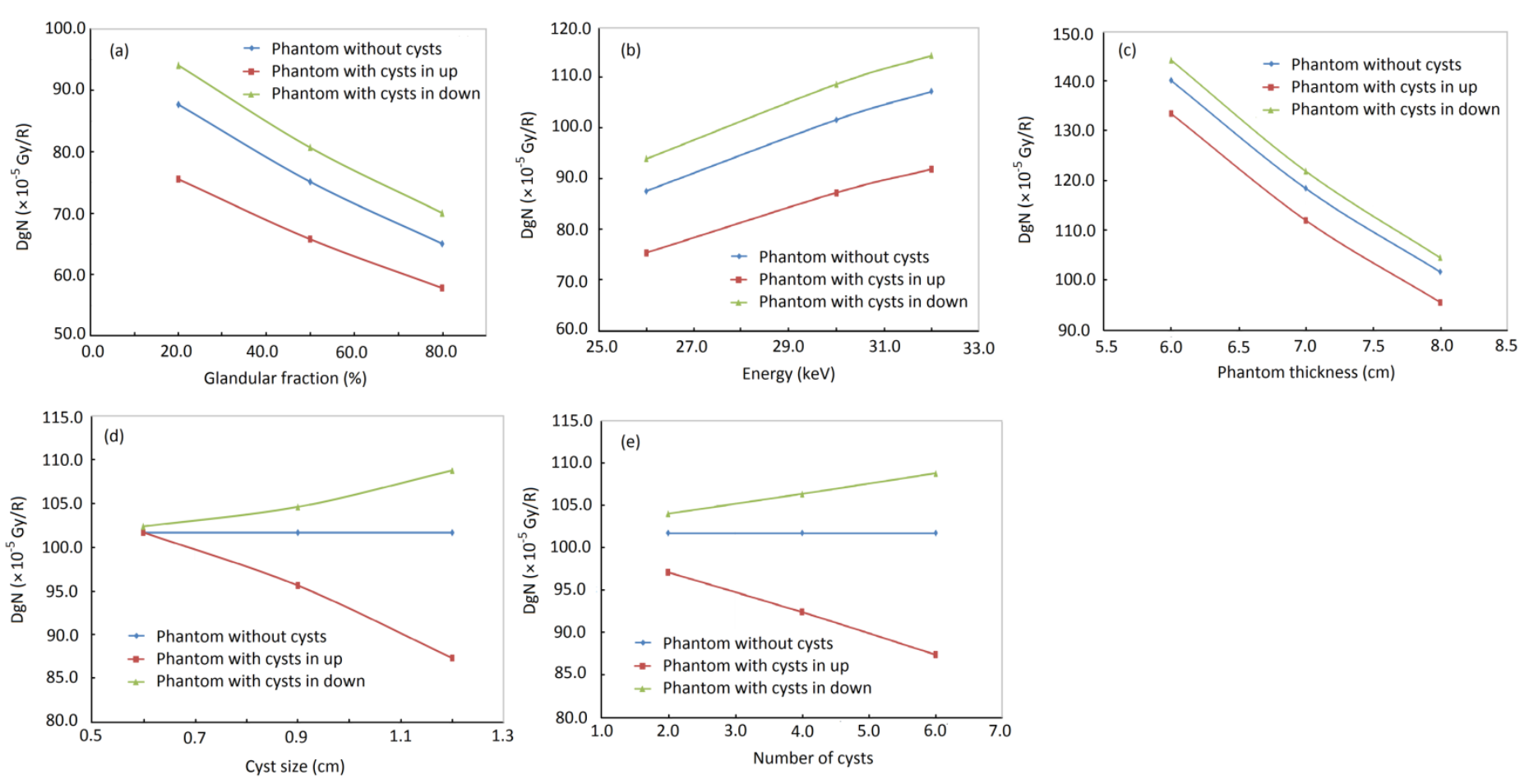

Figure 7. (a) DgN versus the glandular fraction of the breast in the homogeneous breast phantom with and without cysts (all the three phantoms have a height of $8 \mathrm{~cm}$ and the tube voltage was constant $26 \mathrm{kV}$ ) (b) DgN versus the tube voltage in the homogeneous breast phantom with and without cysts (all the three phantoms have a height of $8 \mathrm{~cm}$ and the glandular fraction of the breast phantom was $20 \%$ ) (c) DgN versus the phantom thickness in the homogeneous breast phantom with and without cysts (the $\mathrm{X}$-ray tube voltage applied at all these stages was $30 \mathrm{kV}$ and the radius of the cysts was considered to be the constant value of $0.9 \mathrm{~cm})(\mathrm{d})$ DgN versus the cyst size in the homogeneous breast phantom with and without cysts (the voltage applied at all the stages was $30 \mathrm{kV}$ and all the three phantoms had a glandular fraction of $20 \%$ and thickness of $8 \mathrm{~cm}$ ) (e) DgN versus the cyst number in the homogeneous breast phantom with and without cysts (all the three phantoms had a glandular fraction of $20 \%$ and thickness of $8 \mathrm{~cm}$ and the radius of the cysts and the $\mathrm{X}$-ray tube voltage were $1.2 \mathrm{~cm}$ and $30 \mathrm{kV}$, respectively) 
To evaluate the effect of number of cysts on the $\mathrm{DgN}$, three phantoms were considered, all of them had glandular fraction of $20 \%$ and thickness of $8 \mathrm{~cm}$. The first phantom was considered without any cyst. The second phantom had 6 cysts at depth of $3.1 \mathrm{~cm}$ and the third phantom had 6 cysts at depth of $5.9 \mathrm{~cm}$. The cyst numbers of 2, 4 and 6 were investigated. The radius of the cysts was constant in all the steps and was equal to $1.2 \mathrm{~cm}$. The voltage of the X-ray tube applied at all the steps was $30 \mathrm{kV}$. The results of $\mathrm{DgN}$ values are compared in Figure 7e.

\section{DgN results for heterogeneous breast phantom}

Two phantoms were simulated to investigate the effect of the heterogeneity of breast tissue. The thickness of both phantoms was $8 \mathrm{~cm}$ and the glandular fractions of $0,20,50$, and $100 \%$ were evaluated. The applied voltage for the X-ray tube was 30 $\mathrm{kV}$. The results are shown in Figure 8 .

\section{Discussion}

As it was mentioned earlier, patient dosimetry in mammography imaging is important due to the use of low energy X-rays in mammography and the major energy absorption in breast tissue, as well as the sensitivity of the breast glandular tissue to X-rays. The average glandular dose is the main quantity in mammography patient dosimetry. Various factors can affect $\mathrm{DgN}$. In this study, the effect of these factors, such as the thickness of the breast phantom, glandular fraction, applied X-ray tube voltage, heterogeneity of the glandular tissue and adipose in the breast phantom, and the presence of a cyst in the homogeneous breast tissue on $\mathrm{DgN}$ was investigated.

\section{Validation of X-ray tube simulation}

As it can be seen from Figure 3b, in most data points, these two spectra are in agreement. The peak of the spectrum from the simulation, which represents characteristics X-rays, has a higher height than those of the IPEM spectrum. The IPEM spectrum is based on a semi-empirical method, and as it was discussed in the literature, ${ }^{4}$ this difference in the height of the peaks is also seen in other Monte Carlo studies. To evaluate the accuracy of the simulated spectrum, paired $t$-test was used in the SPSS software. The null hypothesis (no statistically significant difference between the two results) with a $95 \%$ confidence interval was proven and thereby the accuracy of the simulation of the X-ray tube is confirmed.

In order to evaluate the accuracy of the simulation results of the desired mammography machine (Payamed), the PDD was also obtained by two methods of simulation calculations and empirical measurement, and the results are presented in Fig. 4. There is a good agreement between the two graphs and the maximum percentage difference between the two graphs is $5.1 \%$, which is related to the depth of $2 \mathrm{~cm}$.

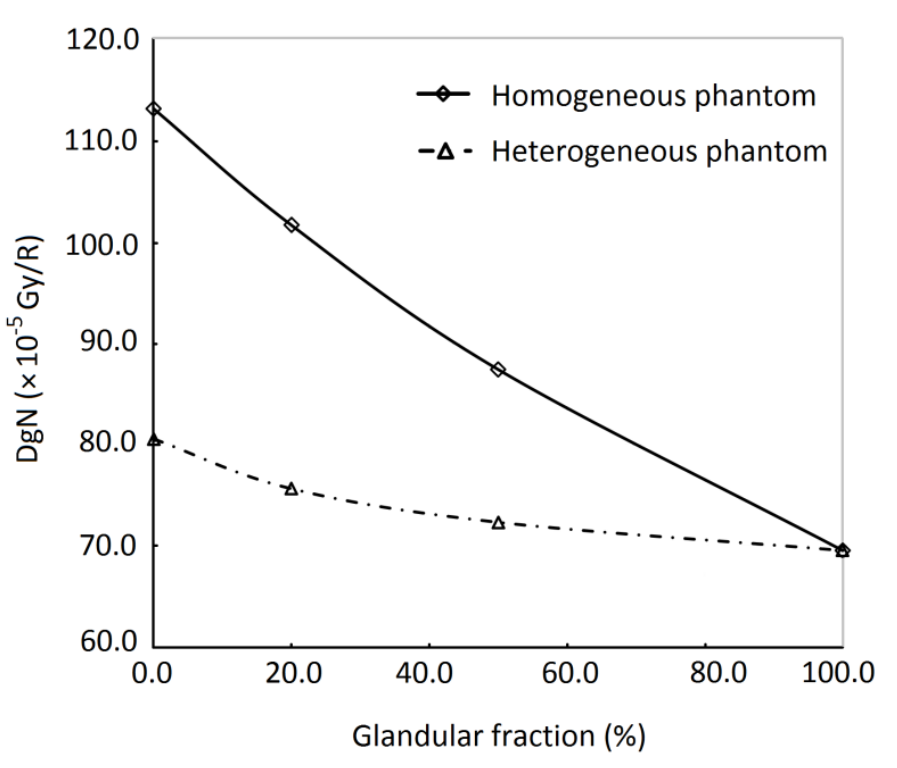

Figure 8. DgN versus the glandular fraction in the homogeneous and heterogeneous breast phantoms (the phantoms had thickness of $8 \mathrm{~cm}$ and the applied voltage for the $\mathrm{X}$-ray tube was $30 \mathrm{kV}$ )

In previous studies, the PDDs were obtained by simulations and experimental methods. ${ }^{11,16}$ In most of these studies, breast equivalent phantoms have been used for experimental measurement. In the study by Niegaprock et al $^{16}$ the results from measurement by thermoluminescent dosimeter and MCNP calculations were compared and the maximum discrepancy of $4 \%$ was reported. In that study, the PDD curve which was obtained by TLD was slightly lower than the simulated PDD curve, especially in steeper depths of the phantom. The discrepancies in the current study are close to that reported in the study by Nigaproke, et al.

\section{DgN in homogeneous breast phantom without cysts}

In this step, the effect of X-ray tube voltage and a glandular fraction of breast were investigated. As it is shown in Figure 5a, with an increase in the glandular fraction of the breast, the $\mathrm{DgN}$ decreases. By increasing the glandular fraction of the breast from 20 to 80 percent, DgN decreases by about 25 percent. This can be attributed to the fact that, with increasing glandular tissue, the underlying layers receive lower doses due to the attenuation of X-rays, although the upper layers of the glandular tissue receive higher doses.

It can be realized from Figure 5b that by increasing the thickness of the breast from 3 to $8 \mathrm{~cm}$, the amount of $\mathrm{DgN}$ decreases by about $60 \%$. This decrease is due to the further attenuation of the X-rays inside the thick phantom than the thin phantom. 


\section{DgN in homogeneous breast phantom in presence of cysts}

Different scenarios were considered for investigating the presence of cysts in the breast phantom. As it is shown in Figure 6, the depth of placement of cysts results in different $\mathrm{DgN}$ values, so that when 6 cysts with a radius of $1.2 \mathrm{~cm}$ are present at depth of $3.1 \mathrm{~cm}, \mathrm{DgN}$ is $14.0 \%$ lower than the $\mathrm{DgN}$ in a phantom without cysts. However, when the same number of cysts are at depth of $5.9 \mathrm{~cm}$ in the breast phantom, $\mathrm{DgN}$ is $6.8 \%$ more than the $\mathrm{DgN}$ value in the phantom without any cyst. This is because that when the cysts are placed at the beginning of the phantom thickness, X-rays are attenuated more and then reached into the glandular tissue. On the other hand, when the cysts are placed at the end of the phantom thickness, it actually replaces the part of the breast tissue that receives the lowest dose, and actually reduces the amount of $\mathrm{DgN}$ in the averaging process. Therefore, placing the cysts in this region increases the average glandular dose and DgN. Of course, the effect of the presence of cysts decreases by decreasing their number and size, as it was shown in Figure 7d and Figure 7e. When the cysts are at the beginning of the phantom and their number is reduced from 6 to 2 , the percentage difference is changed from $14.0 \%$ to $4.8 \%$, and when the radius of cysts is reduced from $1.2 \mathrm{~cm}$ to $0.6 \mathrm{~cm}$, the difference of $14.0 \%$ reaches almost to zero. This means that cysts with a radius less than $0.6 \mathrm{~cm}$ have not effect on the computing the amount of $\mathrm{DgN}$.

As it is shown in Figure 7a, the effect of the glandular fraction of the breast in the homogeneous breast phantom with and without cysts was examined and the results show a slight difference in the calculations. When the cysts are at the beginning of the phantom thickness, and the glandular fraction of the breast is $20 \%$, with the presence of cysts a reduction of $14.0 \%$ is seen in the value of $\mathrm{DgN}$, and when the glandular fraction of the breast is $80 \%$, a reduction of $11.9 \%$ is seen.

According to Figure $\mathbf{7 b}$, there is differences between glandular dose in the homogenous breast phantom and breast phantom with cyst, but these differences almost are the same in various kilovoltages. As it can be seen in Figure 7c, there is differences between glandular dose in the homogenous breast phantom and breast phantom with cyst, but these differences almost are the same in various breast thicknesses.

In general, according to the results, it can be noted that the presence of cysts in breast phantom, with the conditions considered in this modeling, had an effect of $-7 \%$ to $+14 \%$ on $\mathrm{DgN}$ values. This effect is independent of the applied voltage, phantom thickness and phantom glandular percentage, and depends only on the location and depth of the cysts, and their number and size. In cases where cyst accumulation is located at the end of the breast thickness, or the size of the cysts is large or the number of cysts is high, the average glandular dose for the patient is greater than the calculated value by a significant level.
The presence of cysts may influence glandular dose by less than $15 \%$. For calculation of glandular dose for a patient, DgN coefficients should be used, which depend on thickness and composition of the breast. Thickness of breast is normally known with limited accuracy, because the readout for thickness readout is not always ideally calibrated, and compression paddle is not always ideally level to breast support table. Uncertainties of about $15 \%$ in glandular dose calculation may be easily caused by a few millimeters of uncertainty in breast thickness readout. Similarly, the composition of the breast can be assessed either visually or from a breast thickness, with very limited accuracy. Variation of glandular fraction from one category to another may also change glandular dose by $15 \%$. Therefore, while this study shows that glandular fraction and breast thickness has an effect on the determination of breast dose, it should be noted that the determination of these two factors is accompanied by uncertainties and this will have increase the total uncertainty is breast dose determination.

In the present study, Monte Carlo calculations were only performed for certain models of breasts, e.g. certain shapes and dimensions, which are very simplified. This will be incorporated with uncertainties in breast dose determination. Therefore, the glandular dose values presented in this study are only rough estimations of the dose of real patients. In other words, while it is useful to know the influence presence of cysts on breast dose, but the obtained results can not routinely be used for the calculation of correction factors in routine clinical applications.

In the present study, a special mammography unit (PMAM 100-F) was defined, which has a Mo target and a Mo filter. There are also other mammography units with $\mathrm{Mo} / \mathrm{Rh}$ or $\mathrm{W} / \mathrm{Rh}$ target/filter combinations. It is interesting to evaluate the effect of the presence of cysts on dosimetry parameters in the case of such target/filter combinations, and this can be a subject of further studies. In the calculation of $\mathrm{DgN}$, the average glandular dose and air kerma were expressed in terms of Gy and Roentgen, respectively and the units for $\mathrm{DgN}$ is in terms of Gy/R. It should be noted that in the past, exposure (in R) was used instead of air kerma and this method was followed in this research and other similar studies in this field. ${ }^{4,} \mathrm{DgN}$ can also be presented in terms of $\mathrm{mGy} / \mathrm{mGy}$ and conversion of the results from $\mathrm{Gy} / \mathrm{R}$ to $\mathrm{mG} / \mathrm{mGy}$ requires a simple conversion factor. However, this conversion would not have effect on the general trends of the results and the conclusion of this study.

\section{Comparison of DgN values in homogeneous and heterogeneous phantoms}

Figure 8 shows $\mathrm{DgN}$ in terms of the glandular fraction in the homogeneous and heterogeneous breast phantoms. As it is shown in Figure 8, when a heterogeneous breast tissue is assumed, the $\mathrm{DgN}$ value decreases, and this reduction is higher in the case of lower glandular fractions. By increasing the glandular fraction, this difference decreases. The percentage 
difference between the two phantoms (homogeneous and heterogeneous) varies between $0 \%$ to $29 \%$. This result is similar to the results of other studies by researchers. ${ }^{4}$

\section{Conclusion}

In this study, the $\mathrm{DgN}$ value, which is used to calculate the average glandular dose, was taken into account in a homogeneous breast phantom. On the other hand, the breast tissue has a tubular and lipid form. In general, heterogeneities such as fibrotic and fiber-adenoma are seen in the breast tissue that can have an effect on the calculation of the average glandular dose. Each of these factors, can have a measurable effect on the results, and a correction coefficient calculation is proposed for the calculation of $\mathrm{DgN}$ values.

\section{Acknowledgment}

The authors would like to thank Shahid Beheshti University of Medical Sciences for financial support of this study. This study was part of M. Sc. thesis of Mrs. Zeinab Kaveh.

\section{References}

1. World Health Organization. Available at:https://www.who.int/cancer/prevention/diagnosis-screening/breast-cancer/en, Accessed on: January 2019.

2. Dance D, Skinner C, Carlsson G. Breast dosimetry. Appl Radiat Isot. 1999;50(1):185-203. https://doi.org/10.1016/S09698043(98)00047-5

3. Hall EJ, Giaccia AJ. Radiobiology for the radiologist. Lippincott Williams and Wilkins; Philadelphia:222-50. 2006.

4. Boone JM. Glandular breast dose for monoenergetic and high-energy x-ray beams: Monte Carlo assessment. Radiology. 1999;213(1):23-37. https://doi.org/10.1148/radiology.213.1.r99oc3923

5. Stanton L, Villafana T, Day J, Lightfoot D. Dosage evaluation in mammography. Radiology. 1984;150(2):577-84. https://doi.org/10.1148/radiology.150.2.6691119

6. European Commission. European protocol on dosimetry in mammography. Report EUR 16263, EC, Bruxelles, Luxembourg. 1996.

7. Delis H, Spyrou G, Panayiotakis G, Tzanakos G. DOSIS: A Monte Carlo simulation program for dose related studies in mammography. Eur J Radiol. 2005;54(3):371-6. https://doi.org/10.1016/j.ejrad.2004.07.014

8. Bushberg JT, Boone JM. The essential physics of medical imaging. Lippincott Williams \& Wilkins;238-282. 2011.

9. Sookpeng S, Ketted P. Mean glandular dose from routine mammography. Naresuan University J: Sci Technol. 2006:14(3):19-26.

10. Zankl M, Fill U, Hoeschen C, et al. Average glandular dose conversion coefficients for segmented breast voxel models. Radiat Prot Dosimetry. 2005;114(1-3):410-4. https://doi.org/10.1093/rpd/nch513

11. Wu X, Barnes GT, Tucker D. Spectral dependence of glandular tissue dose in screen-film mammography. Radiology. 1991;179(1):143-8. https://doi.org/10.1148/radiology.179.1.2006265

12. Sutter Health (CPMC). Available at: http://www.cpmc.org/services/women/breast/breast_cyst.html. Accessed on: 12 Nov, 2016.

13. American Cancer Society. Available at: https://www.cancer.org/cancer/breast-cancer/non-cancerous-breast-conditions/fibrosis-andsimple-cysts-in-the-breast.html. Accessed on: 12 Nov, 2016.

14. Aznar M, Hemdal B. Absorbed dose measurement in mammography. In: Hayat MA (ed.), Cancer Imaging: Lung and breast carcinomas (Vol. 1), Elsevier; 493-501. 2008. https://doi.org/10.1016/B978-012374212-4.50055-9

15. Dance DR. Monte Carlo calculation of conversion factors for the estimation of mean glandular breast dose. Phys Med Biol. 1990;35(9):1211-9. https://doi.org/10.1088/0031-9155/35/9/002

16. Nigapruke K, Puwanich P, Phaisangittisakul N, Youngdee W. Monte Carlo simulation of average glandular dose and an investigation of influencing factors. J Radiat Res. 2010;51(4):441-8. https://doi.org/10.1269/jrr.10008

17. Hernandez AM, Seibert JA, Boone JM. Breast dose in mammography is about $30 \%$ lower when realistic heterogeneous glandular distributions are considered. Med Phys. 2015;42(11):6337-48. https://doi.org/10.1118/1.4931966

18. Payamed Electronic Industries Co. Available at: http://www.payamed.com/pma100f.asp. Accessed on: 12 Jun, 2017.

19. International Aero Engines. Available at: http://www.iae.it/serie-mammo_23.html. Accessed on: 12 Jun, 2017.

20. Cranley K, Gilmore BJ, Fogarty GWA, Desponds L. Catalogue of diagnostic x-ray spectra and other data. Report No.78, Institute of Physics and Engineering in Medicine - IPEM. 1997.

21. National Institute of Standards and Technology. Available at: https://www.nist.gov/pml/x-ray-mass-attenuation-coefficients. Accessed on: 12 Jun, 2017.

22. Delis H, Spyrou G, Tzanakos G, Panayiotakis G. The influence of mammographic X-ray spectra on absorbed energy distribution in breast: Monte Carlo simulation studies. Radiat Meas. 2005;39(2):149-55. https://doi.org/10.1016/j.radmeas.2004.04.003

23. Rezaei FS. Using Monte Carlo method for evaluation of $\mathrm{kVp}$ and $\mathrm{mAs}$ variation effect on absorbed dose in mammography. European Congress of Radiology, 3 March 2011; Vienna, Austria.

24. Fredenberg E, Dance DR, Willsher P, Moa E, von Tiedemann M, Young KC, et al. Measurement of breast-tissue x-ray attenuation by spectral mammography: first results on cyst fluid. Phys Med Biol. 2013;58(24):8609. https://doi.org/10.1088/0031$9155 / 58 / 24 / 8609$ 